\title{
Does food addiction distinguish a specific subgroup of overweight/obese overeating women?
}

\author{
Catherine Bégin ${ }^{1}$, Marie-Ève St-Louis ${ }^{1}$, Sophie Turmel ${ }^{1}$, Béatrice Tousignant ${ }^{1}$, \\ Louis-Philippe Marion ${ }^{1}$, Francine Ferland ${ }^{2}$, Nadine Blanchette-Martin ${ }^{2}$, \\ Marie-Pierre Gagnon-Girouard ${ }^{1^{*}}$ \\ ${ }^{1}$ School of Psychology, Laval University, Quebec City, Canada; \\ *Corresponding Author: marie-pierre.gagnon-girouard.1@ulaval.ca \\ ${ }^{2}$ Rehabilitation Center for Dependency, Quebec City, Canada
}

Received 15 November 2012; revised 17 December 2012; accepted 24 December 2012

\begin{abstract}
Neurophysiological and behavioral similarities have been evidenced between excessive food consumption leading to obesity and addiction to other substances. In accordance, food addiction was defined following the DSM-IV diagnostic criteria for substance dependence. The aim of this pilot study was to identify a subgroup of women suffering from food addiction $(n=11)$, and to compare them to women suffering from substance-use disorder $(n=23)$, and to women seeking treatment for compulsive overeating but free from food addiction $(n=12)$ on addictionrelated characteristics (reward sensitivity, impulsivity, personality traits, depression, emotion dysregulation). We hypothesized that women with food addiction would be similar to women with substance-use disorders, and different from women with compulsive overeating without food addiction. Participants completed self-reported questionnaires assessing food addiction and other variables related to addiction. Almost half (47.8\%) of women with compulsive overeating fulfilled the criteria for food addiction. Although food addiction does not account for every case of compulsive overeating, it characterizes a specific subgroup of overweight/obese women who show more severe overeating. Women with food addiction seem to be more similar to women suffering from substance-use disorders than to other women with overeating difficulties, particularly regarding impulsivity and self-directedness.
\end{abstract}

Keywords: Food addiction; Compulsive Overeating; Binge Eating; Addiction

\section{INTRODUCTION}

Obesity is a worrisome issue in many countries, and a lot of efforts are devoted toward its understanding and treatment. In Canada, $17.9 \%$ of the population is obese and $33.7 \%$ is overweight, which is similar to what is observed in other occidental countries [1]. Since such a large proportion of the population is overweight or obese, it is clinically impossible to consider it as a uniform condition [2]. In fact, while for some people, excessive food consumption is a passive behavior that occurs without the individual being fully aware of this habit (e.g. frequent snacks, large portions, etc.), for others, overeating is a compulsive behavior that is more obvious to the person but very difficult to control [3].

Recently, it has been suggested by many researchers that compulsive overeating leading to obesity could be conceptualized as an addiction to palatable food (high-fat, -sugar, and -salt food) [2,4,5]. This trend is based on a growing number of empirical evidences demonstrating neurobiological, behavioral, and psychological similarities between compulsive overeating and addiction to alcohol and drugs.

\subsection{Neurobiological, Behavioral, and Psychological Similarities}

Empirical evidences have shown that the pleasure induced by drug or alcohol and the one associated with natural rewards such as palatable food are regulated by the same neural substrates [6]. Notably, both psychoactive and food intake amplify pleasure and enhance mood by increasing extracellular level of dopamine in the mesocorticolimbic pathway, commonly named the "reward system" [2,7-10]. Strongly linked to the mesocorticolimbic pathway, sensitivity to reward plays a major role in addiction and substance-use disorders (SUD) [11,12]. The hypo-dopaminergic hypothesis suggests that individuals with an under-activated reward system are promp- 
ted to use drugs and alcohol, or food, as self-medication to increase their lower capacity to feel pleasure [13] whereas the hyper-dopaminergic hypothesis proposes that individuals with an over-activated reward system feel a higher motivation to repetitively seek pleasurable activities $[2,14,15]$. Both obesity and binge eating have been associated with a strong sensitivity to reward [14, 16].

Impulsivity has also been reported by people with SUD [17] and with compulsive overeating [18,19]. A lesser capacity to plan and to delay gratification leads impulsive individuals to choose immediate pleasure over long-term gains and makes it difficult for them to resist drugs, alcohol or food urges when necessary, which has been observed among SUD patients, binge eaters and obese individuals [20-23].

Previous studies have highlighted other personality traits shared by both populations, such as harm avoidance (the behavioral inhibition of aversive stimuli associated with punishment), novelty seeking (the initiation of appetitive approach in response to novel and pleasurable stimuli) and self-directedness (the capacity to be effecttive and self-confident, and to act in accordance with personal goals) [24-27].

\subsection{Food Addiction Diagnosis Criteria}

In consideration of such numerous similarities, a growing number of experts have proposed that the DSM-IV criteria for substance dependence could be relevant to the concept of food addiction (FA) [4,5]. Moreover, a tool designed to diagnose FA based on these criteria, the Yale Food Addiction Scale, was recently published (YFAS) [28]. The scale was validated among a sample of students who reported a FA prevalence rate of $11.4 \%$. Further support for the FA construct and for the YFAS validity was provided among a sample of obese adults [29]. Results from this study suggest that the YFAS shows a good ability to identify individuals with addictive tendencies toward food. Participants who were classified as having FA (25\%) reported more binge eating, depression, and attention-deficit/hyperactivity than other participants who were age- and weight-paired, as well as more impulsivity, more food cravings, and more emotional eating in the face of a higher affective reactivity [29]. Individuals with FA diagnosed by the YFAS also experience greater food cravings; in particular, they are more convinced that eating can relieve negative emotional states [30]. Finally, when exploring the links between food addiction and psychopathology in a sample of obese patients with binge eating disorder [31], FA, which characterized 56\% of the sample, was positively associated with depression, negative affect, difficulties to regulate emotions, eating disorder symptoms, and negatively with self-esteem. Ta- ken altogether, these evidences suggest that individuals classified as having FA present more difficulties to resist food reward as well as greater affecttive disturbances but poorer strategies to face these difficulties without overeating.

Nonetheless, the only study of FA conducted within a clinical population of obese adults suffering from binge eating disorder [31] did not explore the association of FA with underlying SUD-related characteristics. In addition, this study did not compare FA and non-FA individuals, and no study directly compared individuals with FA to individuals suffering from SUD.

\section{OBJECTIVES AND HYPOTHESIS}

In the context that it seems that not all individuals who overeat could be classified as having FA [2,4], it is necessary to identify the individuals whose eating difficultties could be better described from an addiction perspective. Thus, the general aim of this pilot study was to deepen the understanding of FA among a sample of overweight/obese women seeking treatment for compulsive overeating. The first specific objective was to evaluate the proportion of these women who meet the criteria for FA, as measured by the YFAS. It was expected that this proportion would be higher than what was reported by previous studies among non-psychiatric samples (11.4\% among students in Gearhardt et al. [28]; 25\% among obese individuals in Davis et al. [29]), and comparable to the one observed among obese individuals suffering from binge eating disorder (56.8\%; Gearhardt et al. [31]).

The second specific objective was to compare this particular subgroup of overweight/obese women suffering from FA with (1) women suffering from SUD, and (2) women with compulsive overeating without FA. Groups were compared on psychobiological variables associated with the addiction model, as well as on affective disturbance. It was expected that women reporting FA would be similar to women with SUD and different from women reporting compulsive overeating without FA. Precisely, it was hypothesized that women with SUD and FA would manifest a higher sensitivity to reward, more impulsivity, higher harm avoidance and novelty seeking, and lower self-directedness, as well as higher depressive symptoms and more difficulties to regulate emotions, compared to women with compulsive overeating without FA.

\section{METHOD}

\subsection{Participants}

Two groups of women predominantly Caucasian and aged from 19 to 65 years old were recruited for this study. The first group ( $n=23$ ) was composed of overweight/ obese women (body mass index $>25 \mathrm{~kg} / \mathrm{m}^{2}$ ) seeking 
treatment for compulsive overeating and difficulties to manage weight and free from drug or alcohol dependence. They were recruited at a specialized unit for evaluation and treatment of weight problems at Laval University. Compulsive overeating was confirmed by a clinical interview. Mean age for this group was 41.46 years $(S D=12.53)$. Most women reported having a college or university degree (87.0\%), and half had an annual income superior to $60000 \$(47.6 \%)$. This group was then split, according to the clinical cut-off score of the YFAS score (at least three criteria and the distress/impairment criteria), to identify a subgroup qualifying for FA.

The second group ( $n=27$ ) comprised women currently suffering from SUD (alcohol and/or drug), who were entering treatment in a rehabilitation center (Centre de réadaptation en Dépendence du Québec) at the time of the study. Women from this group were controlled for the presence of FA, and four participants were excluded on the basis of this criterion; analyses were then computed for 23 women in this group. Mean age for this group was 41.65 years $(S D=13.96)$. Half of these women reported having a college or university degree (54.5\%), and $21.7 \%$ had an annual income over 60000\$.

\subsection{Procedure}

Women who volunteered to take part in the research were contacted by phone and a short screening interview was carried out. For women in the overeating group, the SCID was used to make sure they were free of any SUD. Eligible participants were asked to come to the research facility to receive details explanations on the experimenttation and to sign the consent form, approved by the Ethic Committee for Addiction Research (Comité d'Éthique de la Recherche en Toxicomanie). They were weighed and measured in order to calculate their body mass index (BMI), and were asked to complete the whole selfreport questionnaires battery on site or at home. Participants who decided to complete the questionnaires at home were asked to bring them back to the research facility no more than two weeks later. Reminder phone calls were made for participants who did not return their questionnaires after three weeks.

\subsection{Material}

Structured Clinical Interview for DSM-IV Axis I Disorders (SCID-I) [32]. The SCID-I, a semi-structured clinical interview aiming at establishing a diagnosis for DSM-IV Axis I disorders, was administered on the phone to ensure that participants from the overeating group were free of any SUD. According to Zanarini et al. [33], the SUD module shows a good test-retest fidelity $(\kappa=$ 0.71).

Yale Food Addiction Scale (YFAS) [28]. The YFAS, a 25-item self-report questionnaire, is designed to operationalize FA according to the eight criteria for substance dependence presented in the DSM-IV modified for addictive eating behaviors with palatable food (tolerance, withdrawal, loss of control, repeated failed attempts to reduce or stop consumption, extended amount of time devoted to addiction, giving up of important activities, continued use despite physical/psychological problems, clinically significant impairment or distress). There are two scoring options, one that is dichotomous (with or without FA) and one that is a symptom count (zero to seven symptoms). The YFAS presents a good internal consistency ( $\alpha=0.86$ ) and a good validity ( $r=0.46$ à 0.61 ). For the present sample, the Cronbach alpha coefficient was 0.90 .

Binge Eating Scale (BES) [34]. The BE, a 16-item questionnaire, describes both the behavioral (objective) and cognitive (subjective) manifestations of bulimic behaviors among obese individuals. Results have demonstrated that the BES effectively discriminate severe binge eaters from moderate binge eaters and non-binge eaters as diagnosed by clinical interviews. The BES has a sensibility value of 0.85 and a specificity value of 0.20 [35]. For the present sample, the Cronbach alpha coefficient was 0.95 .

Sensitivity to Punishment and Sensitivity to Reward Questionnaire (SPSRQ) [36]. The SPSRQ is a 48-item two-subscale questionnaire measuring aversive and appetitive behavioral systems. Only the sensitivity to reward subscale, referring to the tendency to seek reward stimuli in common situations, was used in the present study. The internal consistency of the French version of the SPSRQ is satisfactory with an alpha de 0.81 [37]. For the present sample, the Cronbach alpha coefficient was 0.89 .

Barratt Impulsiveness Scale (BIS-10) [38]. The BIS10 is a 34-item self-reported questionnaire measuring three dimensions of impulsivity (behavioral impulsivity, cognitive impulsivity, and planning difficulties), along with a global score of impulsivity, which has shown a good internal consistency coefficient of 0.82 [39]. For the present sample, the Cronbach alpha coefficient was 0.78 .

Temperament and Character Inventory (TCI) [40]. This self-reported questionnaire measures four temperament traits (novelty seeking, harm avoidance, reward dependence, and persistence) and three characters traits (self-directedness, cooperativeness, and self-transcenddence). The internal consistency of the French version of the TCI is high, ranging from 0.65 à 0.87 [41]. Only the scores for novelty seeking, harm avoidance and self-directedness were taken into account in the present study. For this sample, the overall Cronbach alpha coefficient was 0.69 .

Depressive Symptoms (BDI-II) [42]. The BDI, a 21- 
item questionnaire, was used to measure depressive symptoms. The BDI-II presents a good internal consistency ( $\alpha=0.92$ in a non-psychiatric population) and a good test-retest reliability (one week: $r=0.93$ ). The Cronbach alpha coefficient in the present sample was 0.94 .

Emotion Dysregulation (DERS) [43]. The DERS is a 36-item self-report questionnaire measuring six different aspects of emotion dysregulation (e.g. non-acceptance, lack of emotional clarity, etc.) as well as a total score of overall difficulties in emotion regulation. Authors reported very good internal consistency $(\alpha=0.93)$ and Cronbach alpha coefficient was 0.96 in the present sample.

\subsection{Statistical Analysis}

All data analyses were performed with the SPSS software (version 13.0). Univariate analyses of variance were performed for age and BMI separately. A multivariate analyse of variance was computed for reward sensitivity, novelty seeking, harm avoidance, self-directedness, and depressive symptoms, followed by Tukey post-hoc tests in order to compare the three groups. The Pillai's trace statistic was used, because it is more robust with small samples. Since their distribution showed heterogeneous variances across groups, both impulsivity and difficulties to regulate emotions were analyzed separately using the Brown-Forsythe test of equality of means and the Games-Howell post-hoc test. Effect sizes (Cohen's $d$ ) of the differences between the FA and the overeating groups were also calculated for dependent variables in order to highlight the differences between women with compulsive overeating and FA and women with compulsive overeating but no FA; an effect size of 0.3 was considered a small effect, 0.5, a medium effect, and 0.8, a large effect [44].

\section{RESULTS}

Concerning the first objective, $47.8 \%$ of overweight/ obese women with compulsive overeating were classified as having FA $(n=11)$. All of these women also presented moderate to severe binge eating, according to the Binge Eating Scale. Half of the women who reported compulsive overeating but no FA $(n=6)$ also showed moderate to severe binge eating while the other half of this subgroup reported mild binge eating $(n=6)$.

Concerning the second objective, means' groups on dependent variables were compared (Table 1). The analyses of variance showed no difference for age, $F(2,42)=$ $0.06, p=0.946$, and a significant difference for BMI, $F$ $(2,27)=8.21, p=0.002$. Women in the FA and the overeating groups were significantly heavier than the SUD group ( $p=0.002 ; p=0.049$ ) but did not differ significantly one from each other $(p=0.600)$. The effect size of the difference between the FA and the overeating
Table 1. Means, standard deviations, and differences between groups on dependent variables.

\begin{tabular}{|c|c|c|c|c|}
\hline & FA & SUD & Overeating & $F$ \\
\hline Age & $\begin{array}{c}40.50 \\
(10.83)\end{array}$ & $\begin{array}{c}41.65 \\
(13.96)\end{array}$ & $\begin{array}{c}42.42 \\
(14.22)\end{array}$ & 0.56 \\
\hline BMI & $\begin{array}{l}31.79 \\
(3.43)\end{array}$ & $\begin{array}{l}24.27 \\
(5.45)\end{array}$ & $\begin{array}{l}34.87 \\
(8.77)\end{array}$ & $8.21^{* *}$ \\
\hline Binge eating & $\begin{array}{l}28.27 \\
(6.29)\end{array}$ & $\begin{array}{c}6.13 \\
(8.78)\end{array}$ & $\begin{array}{l}16.42 \\
(8.14)\end{array}$ & $28.46^{* * *}$ \\
\hline $\begin{array}{l}\text { Sensitivity } \\
\text { to reward }\end{array}$ & $\begin{array}{l}40.64 \\
(5.64)\end{array}$ & $\begin{array}{l}38.57 \\
(9.75)\end{array}$ & $\begin{array}{l}34.58 \\
(8.87)\end{array}$ & 1.47 \\
\hline Impulsivity & $\begin{array}{l}78.00 \\
(5.98)\end{array}$ & $\begin{array}{c}75.83 \\
(12.81)\end{array}$ & $\begin{array}{l}67.58 \\
(5.96)\end{array}$ & $5.50^{*}$ \\
\hline Novelty seeking & $\begin{array}{c}96.36 \\
(33.62)\end{array}$ & $\begin{array}{l}109.13 \\
(29.45)\end{array}$ & $\begin{array}{l}103.33 \\
(24.53)\end{array}$ & 0.72 \\
\hline $\begin{array}{c}\text { Harm } \\
\text { avoidance }\end{array}$ & $\begin{array}{l}105.45 \\
(50.07)\end{array}$ & $\begin{array}{l}110.43 \\
(36.62)\end{array}$ & $\begin{array}{c}86.25 \\
(42.22)\end{array}$ & 1.36 \\
\hline Self-directedness & $\begin{array}{c}69.82 \\
(25.38)\end{array}$ & $\begin{array}{c}96.35 \\
(23.85)\end{array}$ & $\begin{array}{l}112.33 \\
(27.37)\end{array}$ & $8.37^{* * *}$ \\
\hline Depression & $\begin{array}{c}19.27 \\
(13.59)\end{array}$ & $\begin{array}{l}18.48 \\
(9.80)\end{array}$ & $\begin{array}{c}11.25 \\
(10.74)\end{array}$ & 2.06 \\
\hline $\begin{array}{c}\text { Emotion } \\
\text { dysregulation }\end{array}$ & $\begin{array}{c}98.18 \\
(16.03)\end{array}$ & $\begin{array}{l}103.96 \\
(27.10)\end{array}$ & $\begin{array}{c}95.50 \\
(29.86)\end{array}$ & 0.52 \\
\hline
\end{tabular}

group for BMI was $d=0.43$, which is considered a small effect.

The Pillai's trace statistic showed that there were significant differences between groups for the multivariate analyse, $F(12,78)=5.02, p<0.001$. Significant differences between groups were evidenced for binge eating severity ( $p<0.001$ ), impulsivity (Brown-Forsythe test; $F$ $(2,43)=5.50, p=0.008)$, and self-directedness $(p=$ $0.001)$. No difference was found for sensitivity to reward ( $p=0.241)$, novelty seeking ( $p=0.493)$, harm avoidance ( $p=0.267)$, depression ( $p=0.139)$, and difficulties to regulate emotions (Brown-Forsythe test; $F(2,43)=$ 0.518, $p=0.601)$.

As illustrated in Figure 1, post-hoc comparison tests were performed, in order to identify precisely the differences between groups.

First, women from the FA group reported more severe binge eating than women from the two other groups ( $p=$ 0.003; $p<0.001$ ), whereas women from the overeating group showed more severe binge eating than women from the SUD group ( $p=0.003$ ). The effect size of the difference between the FA and the overeating group for binge eating was $d=1.62$, which is considered a large effect.

Concerning addiction-related traits, the FA group was significantly more impulsive than the overeating group 

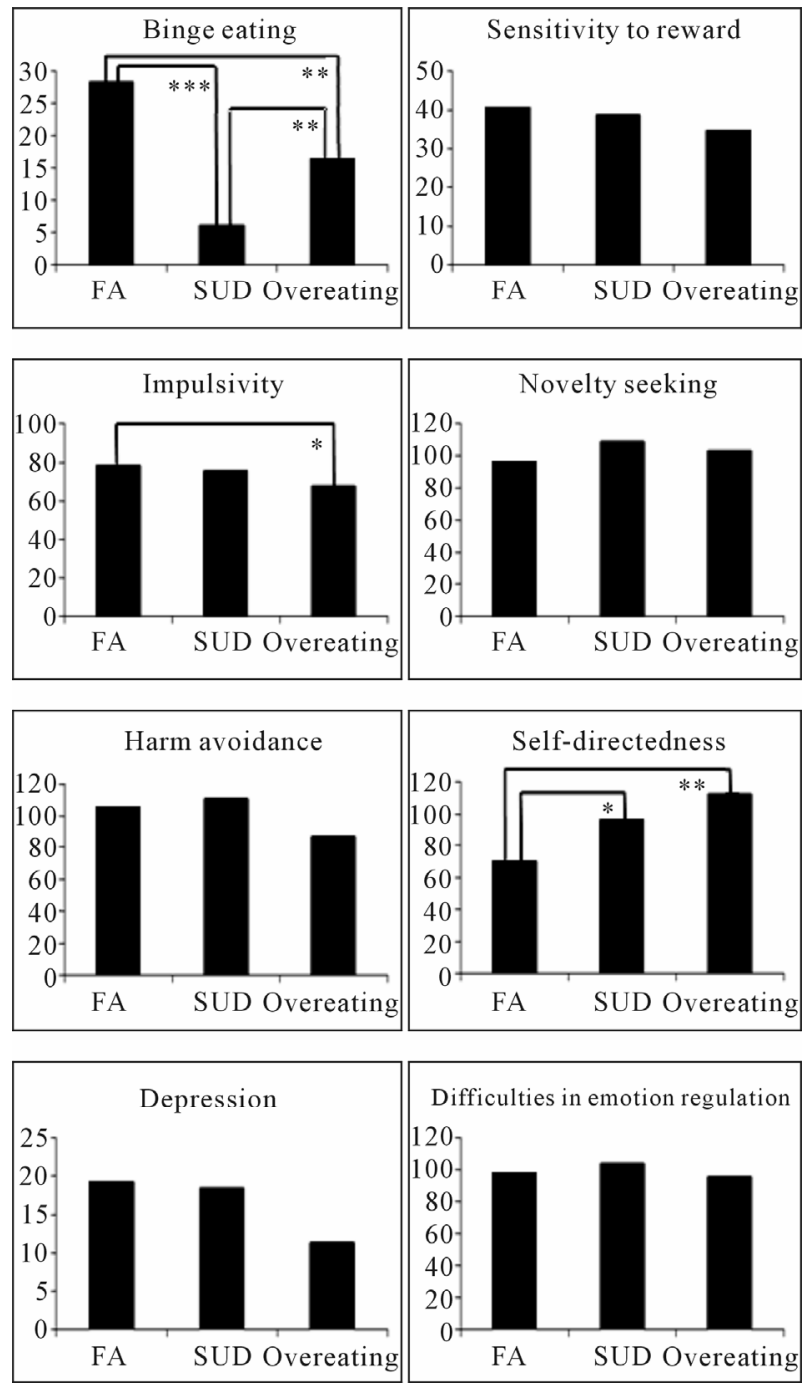

Figure 1. Mean differences between groups. ${ }^{*} p<0.05 ;{ }^{* *} p<$ $0.01 ;{ }^{* * *} p<0.001$. Note: FA = food addiction; SUD = substance-use disorders

( $p=0.040$ ) but was not different from the SUD group ( $p$ $=0.827)$. The effect size of the difference between the FA and the overeating group for impulsivity was $d=1.75$, which is considered a large effect. The FA group was also significantly less self-determined than the overeating group ( $p=0.001$ ) as well as than the SUD group ( $p=$ $0.017)$. The effect size of the difference between the FA and the overeating group was $d=1.61$, which is considered a large effect. Since no significant difference was found for sensitivity to reward, harm avoidance, and novelty seeking, no post-hoc comparisons were computed for these variables. The effect size of the difference between the FA and the overeating group was $d=0.80$ for sensitivity to reward, which is considered a large effect, $d$ $=0.42$ for harm avoidance, which is considered a small effect, and $d=0.24$ for novelty seeking, which does not reach the magnitude of a small effect.
As well, no post-hoc comparison was computed for affective reactivity variables. The effect size of the difference between the FA and the overeating group was $d=$ 0.66 for depression, which is considered a moderate effect, and $d=0.42$ for difficulties to regulate emotions, which is considered a small effect.

\section{DISCUSSION}

Based on previous empirical evidences concerning similarities of FA and addiction to other substances such as alcohol and drugs, the main aim of the study was to deepen the understanding of the subgroup of women suffering from FA among a population of overweight/obese women seeking treatment for compulsive overeating. The FA subgroup was compared to women with current SUD and to overweight/obese women suffering from compulsive overeating without FA.

As hypothesized, almost half of the overweight/obese women suffering from compulsive overeating reached the threshold for FA, which is a considerably larger proportion than what was previously reported among students and obese individuals from the community $[28,29]$ and similar to what was observed among obese binge eaters [31]. On one hand, this result suggests that FA seems to characterize a significant number of overweight/obese individuals seeking treatment for overeating problems. Results also confirm previous findings from Davis and her colleagues [29] and Gearhardt and her colleagues [31] by showing that these women showed more severe eating pathology than women without FA. On the other hand, they also suggest that FA does not account for all cases of compulsive overeating since half of our subgroup of overweight/obese women who did not fulfill the criteria for FA presented moderate to severe binge eating $(n=6)$. These results highlight both the importance of considering FA as a clinically relevant construct and the importance of effectively detecting the individuals who are characterized by this disorder and those who are not.

Findings also suggest that, in general, the subgroup of women suffering from FA was more similar to women suffering from SUD than to other overweight/obese women with compulsive overeating who do not suffer from FA. Even though the sample of this pilot study was small, some significant differences between groups and several moderate to very large effect sizes were observed, which seems to highlight a specific pattern of results. First, aside from a significant difference on self-directedness (where the FA group showed an even lower score than the SUD group), the FA group did not differ from the SUD group. Second, women in the FA group distinguish themselves from women in the overeating group on both impulsivity and self-directedness. Large effect sizes were evidenced for these variables as well as for sensitivity to 
reward. Present findings demonstrate that these characteristics seem to be more related to an addiction disorder and not a core feature of compulsive overeating per se. Women with FA seems to be less able than overweight/ obese women without FA to refrain themselves when it would be necessary, they do not believe that they are able to fix themselves goals and achieve them, and they seems to be more incline to seek and choose short-term pleasurable experiences over more beneficial long-term choices. In that way, they are similar to women suffering from SUD, even sometimes reporting higher scores than them. These characteristics may put them at risk to be less able to stop or to avoid overeating as well as to plan, implement and maintain behavioral changes which may provoke or exacerbate their distress and lower even more their self-directedness.

No differences between the FA and the overeating group were observed on novelty seeking and harm avoidance. In fact, the effect size of the difference between the FA group and the overeating group for novelty seeking did not even reach a small effect and the one for harm avoidance was small, which suggests that there is really no difference between groups on these variables. This result is coherent with Fassino and colleagues' findings [24], since, in their sample, treatment-seeking obese individuals were not different from lean participants neither on novelty seeking, nor on harm avoidance. Treatment-seeking obese women, such as participants in our sample, may be less sensitive to novelty and to punishment than obese individuals from the community.

Finally, concerning affective reactivity, women from the FA group seem to be more depressed $\left(\mathrm{M}_{\mathrm{BDI}}=19.27 \pm\right.$ 13.59) than women from the overeating group $\left(\mathrm{M}_{\mathrm{BDI}}=\right.$ $11.25 \pm 10.74)$, but this difference failed to reach statistical significance. However, the medium effect size of the difference between groups suggests that this difference could have been evidenced in a larger sample, as was the case in Gearhardt and colleagues [31]. Yet, even though women suffering from FA seem to report higher depressive symptoms than women suffering from overeating, they do not show greater difficulties to regulate emotions. There is a considerable amount of evidence that emotion dysregulation is associated with compulsive overeating, suggesting that overeating is a strategy to avoid or to be distracted from unwanted negative affect [45]. Yet, results suggest that emotion dysregulation is an issue that characterizes compulsive overeaters in general, and not only the subgroup of these women who also suffers from FA.

Some important limitations must be taken into account when interpreting these results. First, the sample size was relatively small, and most differences between groups did not reach statistical significance. Yet, for several of these differences, effect sizes were moderate or large, suggest- ing that substantial differences existed between groups and could have been evidenced in larger samples. For example, an a posteriori power analysis revealed that both groups should have been formed of at least 35 participants in order to detect significant differences concerning sensitivity to reward. Second, even though participants in the FA and the overeating group all exhibit clinically relevant compulsive overeating, the instruments used in the present study did not allow diagnosing formal binge eating disorders among them. Third, most instruments measuring addiction-related variable were general and did not specifically concern food and eating. For example, the sensitivity to reward scale focuses on different rewards such as seeking financial gains: women who suffer from FA may be more sensitive to food reward in particular but not as sensitive to other rewards, which may have lessen the differences between groups. It would be interesting to replicate these results with food-related instruments.

Taken altogether, these results add to previous evidences by demonstrating that FA characterizes a specific subgroup of overweight/obese women seeking treatment for compulsive overeating. These findings also give additional support to the validity of the FA construct by showing that overweight/obese women with FA (1) distinguish themselves from other overweight/obese women with compulsive overeating but no FA, and (2) are not different from women suffering of SUD. It may suggest that previously observed similarities between individuals suffering from SUD and obese individuals could in fact be attributable to the specific subgroup of individuals for whom obesity may be the result of an addiction to palatable food. These results justify the importance of detecting the presence of FA among overweight/obese women seeking treatment for eating disturbances as well as the relevance of developing a FA treatment, inspired from SUD approaches, in order to help these individuals to learn to regulate their impulsivity and their sensitivity to reward as well as to develop their self-directedness and decision-making capacities.

\section{REFERENCES}

[1] Statistique Canada (2010) Product \# 82-228-XWF in the manual of Statistic Canada. http://www12.statcan.gc.ca/health-sante/82-228/index.cf m?Lang=F

[2] Davis, C. and Carter, J.C. (2009) Compulsive overeating as an addiction disorder. A review of theory and evidence. Appetite, 53, 1-8. doi:10.1016/j.appet.2009.05.018

[3] Drewnowski, A. and Darmon, N. (2005) The economics of obesity: dietary energy density and energy cost. American Journal of Clinical Nutrition, 82, 265S-273S.

[4] Gearhardt, A.N., Corbin, W.R. and Brownell, K.D. (2009) 
Food addiction: An examination of the diagnostic criteria for dependence. Journal of Addiction Medicine, 3, 1-7. doi:10.1097/ADM.0b013e318193c993

[5] Volkow, N.D. and O’Brien, C.P. (2007) Issues for DSM$\mathrm{V}$ : Should obesity be included as a brain disorder? American Journal of Psychiatry, 164, 708-710. doi:10.1176/appi.ajp.164.5.708

[6] Pelchat, M.L. (2002) Of human bondage: Food craving, obsession, compulsion, and addiction. Physiology \& Behavior, 76, 347-352. doi:10.1016/S0031-9384(02)00757-6

[7] Hernandez, L. and Hoebel, B.G. (1988) Food reward and cocaine increase extracellular dopamine in the nucleus accumbens as measured by microdialysis. Life Sciences, 42, 1705-1712. doi:10.1016/0024-3205(88)90036-7

[8] Kelley, A.E., Baldo, B.A., Pratt, W.E. and Will, M.J. (2005) Corticostriatal-hypothalamic circuitry and food motivation: Integration of energy, action and reward. Physiology \& Behavior, 86, 773-795. doi:10.1016/j.physbeh.2005.08.066

[9] Martel, P. and Fantino, M. (1996) Influence of the amount of food ingested on mesolimbic dopaminergic system activity: A microdialysis study. Pharmacology, Biochemistry \& Behavior, 55, 297-302. doi:10.1016/S0091-3057(96)00087-1

[10] Wang, G.J., Volkow, N.D., Thanos, P.K. and Fowler, J.S. (2004) Similarity between obesity and drug addiction as assessed by neurofunctional imaging: A concept review. Journal of Addiction Disorders, 23, 39-53. doi:10.1300/J069v23n03_04

[11] Volkow, N.D., Wang, G.J., Fowler, J.S., Logan, J., Gatley, S.J., Gifford, A., Hitzemann, R., Ding, Y.S. and Pappas, N. (1999) Prediction of reinforcing responses to psychostimulants in human by brain dopamine D2 receptor levels. American Journal of Psychiatry, 156, 1440-1443.

[12] Volkow, N.D., Wang, G.J., Fowler, J.S., Logan, J., Gatley, S.J., Wong, C., et al. (1999) Reinforcing effects of psychostimulants in humans are associated with increases in brain dopamine and occupancy of $\mathrm{D}(2)$ receptors. The Journal of Pharmacology and Experimental Therapeutics, 291, 409-415.

[13] Blum, K, Braverman, E.R., Holder, J.M., Lubar, J.F., Monastra, V.J., Miller, D., et al. (2000) Reward deficiency syndrome: A biogenetic model for the diagnosis and treatment of impulsive, addictive, and compulsive behaviors. Journal of Psychoactive Drugs, 32, 1-112.

[14] Davis, C., Strachan, S. and Berkson, M. (2004) Sensitivity to reward: Implications for overeating and overweight. Appetite, 42, 131-138. doi:10.1016/j.appet.2003.07.004

[15] Loxton, N.J. and Dawe, S. (2006) Reward and punishment sensitivity in dysfunctional eating and hazardous drinking in women: Associations with family risks. Appetite, 47, 361-371. doi:10.1016/j.appet.2006.05.014

[16] Davis, C. and Woodside, D.B. (2002) Sensitivity to the rewarding effects of food and exercise in the eating disorders. Comprehensive Psychiatry, 43, 189-194. doi:10.1053/comp.2002.32356

[17] Dawe, S. and Loxton, N.J. (2004) The role of impulsivity in the development of substance use and eating disorders. Neuroscience \& Biobehavioral Reviews, 28, 343-351. doi:10.1016/j.neubiorev.2004.03.007

[18] Galanti, K., Gluck, M.E. and Geliebter, A. (2007) Test meal intake in obese binge eaters in relation to impulsiveity and compulsivity. International Journal of Eating Disorders, 40, 727-732. doi:10.1002/eat.20441

[19] Guerrieri, R., Nederkoorn, C. and Jansen, A. (2007) How impulsiveness and variety influence food intake in a sample of healthy women. Appetite, 48, 119-122. doi:10.1016/j.appet.2006.06.004

[20] Davis, C., Levitan, R.D., Muglia, P., Bewell, C. and Kennedy, J.L. (2004) Decision-making deficits and overeating: A risk model for obesity. Obesity Research, 12, 929935. doi:10.1038/oby.2004.113

[21] Davis, C., Patte, K., Curtis, C. and Reid, C. (2010) Immediate pleasures and future consequences. A neuropsychological study of binge eating and obesity. Appetite, $\mathbf{5 4}$ 208-213. doi:10.1016/j.appet.2009.11.002

[22] Vassileva, J., Petkova, P., Georgiev, S., Martin, E.M., Tersiyski, R., Raycheva, M., et al. (2007) Impaired decisionmaking in psychopathic heroin addicts. Drug \& Alcohol Dependence, 86, 287-289. doi:10.1016/j.drugalcdep.2006.06.015

[23] Verdejo-Garcia, A., Benbrook, A., Funderburk, F., David, P., Cadet, J.L. and Bolla, K.I. (2007) The differential relationship between cocaine use and marijuana use on decision-making performance over repeat testing with the Iowa Gambling Task. Drug \& Alcohol Dependence, 90, 2-11. doi:10.1016/j.drugalcdep.2007.02.004

[24] Fassino, S., Leombruni, P., Piero, A., Daga, G.A., Amianto, F., Rovera, G., et al. (2002) Temperament and character in obese women with and without binge eating disorder. Comprehensive Psychiatry, 43, 431-437. doi:10.1053/comp.2002.35906

[25] Hosak, L., Preiss, M., Halir, M., Cermakova, E. and Csemy, L. (2004) Temperament and character inventory (TCI) personality profile in metamphetamine abusers: A controlled study. European Psychiatry, 19, 193-195. doi:10.1016/j.eurpsy.2004.04.003

[26] Le Bon, O., Basiaux, P., Streel, E., Tecco, J., Hanak, C., Hansenne, M., et al. (2004) Personality profile and drug of choice; a multivariate analysis using Cloninger's TCI on heroin addicts, alcoholics, and a random population group. Drug \& Alcohol Dependence, 73, 175-182. doi:10.1016/j.drugalcdep.2003.10.006

[27] Sullivan, S., Cloninger, C.R., Przybeck, T.R. and Klein, S. (2007) Personality characteristics in obesity and relationship with successful weight loss. International Journal of Obesity, 31, 669-674.

[28] Gearhardt, A.N., Corbin, W.R., Brownell, K.D. (2009) Preliminary validation of the Yale Food Addiction Scale. Appetite, 52, 430-436. doi:10.1016/j.appet.2008.12.003

[29] Davis, C., Curtis, C., Levitan, R.D., Carter, J.C., Kaplan, A.S. and Kennedy, J.L. (2011) Evidence that "food addiction" is a valid phenotype of obesity. Appetite, 57, 711-717. doi:10.1016/j.appet.2011.08.017

[30] Meule, A. and Kubler, A. (2012) Food cravings in food 
addiction: The distinct role of positive reinforcement. Eating Behaviors, 13, 252-255.

doi:10.1016/j.eatbeh.2012.02.001

[31] Gearhardt, A.N., White, M.A., Masheb, R.M., Morgan, P.T., Crosby, R.D. and Grilo, C.M. (2012) An examination of the food addiction construct in obese patients with binge eating disorder. International Journal of Eating Disorders, 45, 657-663. doi:10.1002/eat.20957

[32] First, M.B., Spitzer, R.L., Gibbon, M. and Williams, J.B. (2002) Structural Clinical Interview for Dsm-Iv-Tr Axis I Disorders, Research Version, Patient Edition (SCID-I/P). Biometrics Research, New York State Psychiatric Institute, New York.

[33] Zanarini, M.C., Skodol, A.E., Bender, D., Dolan, R., Sanislow, C., Schaefer, E., et al. (2000) The collaborative longitudinal personality disorders study: Reliability of axis I and II diagnoses. Journal of Personality Disorders, 14, 291-299. doi:10.1521/pedi.2000.14.4.291

[34] Gormally, J., Black, S., Daston, S. and Rardin, D. (1982) The assessment of binge eating severity among obese persons. Addictive Behaviors, 7, 47-55. doi:10.1016/0306-4603(82)90024-7

[35] Marcus, M. and Wing, R.R. (1983) Binge eating and dietary restraint. Annual Meeting of the Society of Behavioral Medicine, Baltimore, 4 March 1983.

[36] Torrubia, R., Àvila, C., Moltò, J. and Caseras, X. (2001) The sensitivity to punishment and sensitivity to reward questionnaire (SPSRQ) as a measure of Gray's anxiety and impulsivity dimensions. Personality and Individual Differences, 31, 837-862. doi:10.1016/S0191-8869(00)00183-5

[37] Lardi. C., Billieux, J., d'Acremont, M. and Van Der Linden, M. (2008) A French adaptation of a short version of the Sensitivity to Reward Questionnaire (SPSRQ). Personality and Individual Differences, 45, 722-725.

doi:10.1016/j.paid.2008.07.019
[38] Patton, J.H., Stanford, M.S. and Barratt, E.S. (1995) Factor structure of the Barratt Impulsiveness Scale. Journal of Clinical Psychology, 51, 768-774. doi:10.1002/1097-4679(199511)51:6<768::AID-JCLP22 70510607>3.0.CO;2-1

[39] Bayle, F.J., Bourdel, M., Caci, H., Gorwood, P., Chignon, J. and Ades, J. (2000) Factorial structure of the translation of the Barratt impulsive scale (BIS-10) Canadian Journal of Psychiatry, 45, 156-166.

[40] Cloninger, C.R., Svrakic, D.M. and Przybeck, T.R. (1993) A psychobiological model of temperament and character. Archives of General Psychiatry, 50, 975-990. doi:10.1001/archpsyc.1993.01820240059008

[41] Chakroun-Vinciguerra, N., Faytout, M., Pélissolo, A. and Swendsen, J. (2005) French validation of the short version of the temperament and character inventory (TCI125). Journal of Cognitive and Behavioral Therapy, 15, 27-33. doi:10.1016/S1155-1704(05)81209-1

[42] Beck, A.T., Steer, R.A. and Brown, G.K. (1996) BDI-II manuel. 2nd Edition, Psychological corporation, San Antonio.

[43] Gratz, K.L. and Roemer, L. (2004) Multidimensional assessment of emotion regulation and dysregulation: Development, factor Structure, and initial validation of the difficulties in emotion regulation scale. Journal of Psychopathology and Behavioral Assessment, 26, 41-54. doi:10.1023/B:JOBA.0000007455.08539.94

[44] Cohen, J. (1988) Statistical power analysis for the behavioral sciences. 2nd Edition, Lawrence Erlbaum Associates, Hillsdale.

[45] Whiteside, U., Chen, E., Neighbors, C., Hunter, D., Lo, T. and Larimer, M. (2007) Difficulties regulating emotions: Do binge eaters have fewer strategies to modulate and tolerate negative affect? Eating Behaviors, 8, 162-169. doi:10.1016/j.eatbeh.2006.04.001 